\title{
Training and well-equipped facility increases the odds of skills of health professionals on helping babies breathe in public hospitals of Southern Ethiopia: cross- sectional study
}

\author{
Abera Mersha $^{1 *}$ (D), Shitaye Shibiru ${ }^{1}$, Teklemariam Gultie $^{2}$, Nega Degefa ${ }^{1}$ and Agegnehu Bante ${ }^{1}$
}

\begin{abstract}
Background: Health professionals equipped with the adequate skills of helping baby breath remain the backbone in the health system in improving neonatal outcomes. However, there is a great controversy between studies to show the proximate factors of the skills of health care providers in helping babies breathe. In Ethiopia, there is a paucity of evidence on the current status of health care provider's skills of helping babies breathe despite the improvement in neonatal health care services. Therefore, this study intends to fill those gaps in assessing the skills of helping babies breathe and its associated factors among health professionals in public hospitals in Southern Ethiopia.

Methods: A facility-based cross-sectional study was conducted among 441 health professionals from March 10 to 30, 2019. A simple random sampling method was used to select the study participants. The data were collected through pre-tested interviewer-administered questionnaire and observational checklist. A binary logistic regression model was used to identify significant factors for the skills of helping babies breathe by using SPSS version 25. The $P$-value $<0.05$ used to declare statistical significance.

Results: Overall, $71.1 \%$ (95\%Cl: $66.2,75.4 \%)$ of health professionals had good skills in helping babies breathe. Age group from 25 to $34(\mathrm{AOR}=2.24 ; 95 \% \mathrm{Cl}: 1.04,4.81)$, training on helping babies breathe $(\mathrm{AOR}=2.69 ; 95 \% \mathrm{Cl}: 1.49$, 4.87), well-equipped facility ( $\mathrm{AOR}=2.15 ; 95 \% \mathrm{Cl}: 1.09,4.25)$, and adequate knowledge on helping babies breathe $(A O R=2.21 ; 95 \% C l: 1.25,3.89)$ were significantly associated with a health professionals good skill on helping babies breathe.

Conclusions: Even though a significant number of care providers had good skills in helping babies breathe, yet there is a need to further improve the skills of the provider in helping babies breathe. Hence, health facilities should be equipped with adequate materials and facilitate frequent training to the provider.
\end{abstract}

Keywords: Helping babies breathe, Neonatal resuscitation, Management of neonatal complications

\footnotetext{
*Correspondence: mershaabera@gmail.com

'Department of Nursing, College of Medicine and Health Sciences, Arba

Minch University, Arba Minch, Ethiopia

Full list of author information is available at the end of the article
}

(c) The Author(s). 2019 Open Access This article is distributed under the terms of the Creative Commons Attribution 4.0 International License (http://creativecommons.org/licenses/by/4.0/), which permits unrestricted use, distribution, and reproduction in any medium, provided you give appropriate credit to the original author(s) and the source, provide a link to the Creative Commons license, and indicate if changes were made. The Creative Commons Public Domain Dedication waiver (http://creativecommons.org/publicdomain/zero/1.0/) applies to the data made available in this article, unless otherwise stated. 


\section{Background}

Achieving the Sustainable Development Goal (SDG) targets for ending preventable mortality and the provision of universal health coverage will require large-scale approaches to improving the quality of health care [1]. Globally, significant improvement has been registered in reducing under-five mortality. However, a reduction in neonatal death remains stagnant particularly in subSaharan Africa [2, 3].

Neonatal mortality accounts for an increasing proportion of mortality in under-five children worldwide. Most newborn deaths befall in low and middle-income countries. The majority of neonatal mortality are found in sub-Saharan Africa [4, 5]. Birth asphyxia causes approximately 27 to $30 \%$ of neonatal deaths in resource-limited countries [6]. By providing quality care during delivery and the postpartum period, around $2 / 3$ of the world's neonatal mortality may be prohibited $[7,8]$.

Countrywide, great achievement has been marked by the application of helping babies breathe (HBB) [8]. After delivery, newborns who are breathing and crying may have waited at least for one to three minutes for cord clamping, but for those who are not, a cord is clamped immediately and resuscitation initiated [9]. Helping babies breathe is a set of interventions used to assist the airway, breathing, and circulation ( $\mathrm{ABC}$ of life) of a newborn following the birth or to help it breathe and to help its heart beat $[10,11]$. Infants who required face mask ventilation (FMV) were more likely to die predominantly when the intervention was not given on time or sustained [12].

Health care providers are responsible for HBB. During resuscitation majority of newborns respond with simple steps, such as drying, stimulation, warmth, and bag-mask ventilation $[13,14]$. Effective application of neonatal resuscitation for those in need is identified squandered occasions to provide lifesaving care and highlighted the need to improve the accessibility of indispensable apparatus and ensure that birth attendants acquire and maintain their skills to provide high-quality newborn care [15].

Accessibility and utility of different apparatus, and having skillful personnel is very vital to comprehend the advantage from HBB [7, 8]. Good skill of health professionals on the HBB is essential for improving the neonatal outcome. It has an inordinate upshot to identify, manage, and avoid unwanted outcomes [16, 17]. Based on the evidence, health professionals have the supremacy to improve the excellence of care in the health care delivery system [18].

The success of HBB depends on the provider's clinical skill as well as how to perform essential steps and how to access basic HBB equipment. Report from studies done in Africa and Asian countries showed that welltrained health professionals on the $\mathrm{HBB}$ are not steadily available in all health care facilities $[19,20]$. Studies conducted in Lake Zone and 12 regions of Tanzania stated that health care provider's skills about helping babies breathe were 32.4 , and $0.03 \%$ respectively $[21,22]$. Similarly, a study in Cameron showed health care providers were competent at providing essential newborn care, but they lacked skills for proper handling of newborns that do not breathe at birth, only $24 \%$ of tasks [23]. In Nigeria, $97 \%$ of delivery attendants do not execute the competencies on resuscitation [15]. The overall skills of midwives, nurses, pediatrics residents, and OB-GYN residents were insufficient as shown in a study done in Ethiopia, the overall mean was $6.8(\mathrm{SD}=3.9)$ [24].

Lack of professional support and infrequent resuscitation skills practice are commonly cited as barriers to skill retention after $\mathrm{HBB}$ training [25]. The working facility, experience, limited provider knowledge, inadequate training, and poor availability of equipment were determinants of health professional skill in newborn resuscitation [13, 22, 23, 26, 27].

Skills learned in standardized courses are estimated to last only a few months. Neonatal Resuscitation Program skills or HBB deteriorates immediately after certification. Significant skill deficits were seen at baseline raising concerns regarding the efficacy of the current course structure. Discrepancies in knowledge and skill retention may impact caregiver performance [28].

There is a great controversy between studies to show the significant factors that affect the skills of health care providers. Some studies did not assess the most proximal factors of skills on HBB like facility-related factors. But other studies revealed a trivial association and does not assess the skills of health care providers as well as their association. Besides, there is a scantiness of information's in Ethiopia that shows the skills about HBB, despite the improvement in neonatal health care services to the knowledge of the investigator. Therefore, this study fills those gaps by assessing the current skills of $\mathrm{HBB}$ and factors affecting in the hospitals of Gamo, Gofa, Segen Areas People, Konso and South Omo Zone, Southern Ethiopia.

\section{Methods}

\section{Study setting and period}

This study was conducted in the Hospitals of Gamo, Gofa, Segen Areas People, Konso and South Omo Zone, Ethiopia from March 10-30, 2019. Gamo, Gofa, Segen Areas People, Konso and South Omo area are administrative Zones in the Southern part of Ethiopia. Those Zones hosted different general and primary hospitals, which serve the community by providing preventive and curative services. There are five functional hospitals in the Gamo Zone (Arba Minch General Hospital, Chencha Primary Hospital, Kamba Primary Hospital, Gerese Primary Hospital and Selamber Primary 
Hospital), one hospital in Gofa Zone (Sawla General Hospital), two hospitals in Segen Areas People Zone (Gidole Primary Hospital and Amaro Kele Primary Hospital), one hospital in Konso Zone (Karat Primary Hospital) and two hospitals in South Omo Zone (Jinka General Hospital and Gazer Primary Hospital).

\section{Study design}

The facility-based cross-sectional study design was employed.

\section{Population}

\section{Source population}

The source population was all health professionals who were working in Hospitals of Gamo, Gofa, Segen Areas People, Konso and South Omo Zone, Southern Ethiopia.

\section{Study population}

The study population was all health professionals who were working on delivery, neonatal intensive care unit $(\mathrm{NICU})$, pediatric wards and operating room (OR) in selected Hospitals.

\section{Inclusion and exclusion criteria}

All health professionals who were staff in the respective wards in each hospital were included in this study, whereas those health professionals on annual leave at the time of data collection were excluded from this study.

\section{Sample size determination}

The sample sizes for all objectives were computed using Epi info 7 stat Calc. For the first specific objective (to assess the skills on helping babies breathe) single population proportion was used by considering the following assumption: $P=0.475$ from the study conducted in Ethiopia [24], 95\% level of confidence and 5\% margin of error. To determine the sample size for the 2nd objective (to identify associated factors) two-sample comparison proportions were used. The sample size of the 1st objective was greater than that of the 2 nd objective. So, a non-response rate of $15 \%$ was added to the sample size of the 1st objective $(n=383)$ to come up with the final sample size. As a result, the calculated sample size for this study was 441 .

\section{Sampling techniques}

There are eleven fully functional hospitals in five Zones of Southern Ethiopia (Gamo, Gofa, Segen Areas People, Konso and South Omo Zone). A simple random sampling method was used to select nine hospitals from them. Initially, the calculated sample size was proportionally allocated to each hospital based on the number of health professionals who were working on the respective wards of each hospital. Secondly, a table of the random number was used to select each health professionals based on proportions to come up with the calculated sample size (Fig. 1).

\section{Data collection tools}

A pre-tested structured both self and interview administered questionnaires and observational checklist were used to collect the data. Some tools were developed or adapted from different works of literature and guidelines. But, to measure the outcome variables, a standard questionnaire was used which were developed, reviewed, revised and approved in different workshops [22, 29-32]. The tool contains five main parts: basic characteristics, provider related questions, facility assessment tools (observational checklist and interview), knowledge assessment tools and skill assessment tools. The pre-test was carried out with $5 \%$ of study subjects in other health care institutions and necessary modifications and amendments were taken accordingly before actual data collection (Additional files 1 and 2).

\section{Data collectors and data collection procedures}

A well-trained three BSc holder nurses and six midwives for data collection, and three MSc holder nurse for supervision were involved. The data were collected from health professionals in nine selected hospitals, which were found in five zones of Southern Ethiopia. The data collectors were given information about the study aim and the possible procedures for study participants before providing the questionnaire and interviewing the study participants. After stating the study aim, the data collectors offered a self-administered questionnaire to assess the knowledge of helping babies breathe. Then, each study, participants were interviewed for the skills of helping babies breathe in the room which was arranged by data collectors during an off-work time after returning the self-administered questionnaire. In the end, key informants were interviewed and observational checklists were used to assess the hospitals for the availability of guidelines, essential items, and infrastructures.

\section{Study variables}

Health professional's skills of HBB were the dependent variable, and socio-demographic characteristics, providerrelated factors, facility-related factors, and knowledge on HBB were independent variables for this study.

\section{Measurements}

The description and measurements of the outcome and some explanatory variables used in this study were stated in detail below (Table 1). 


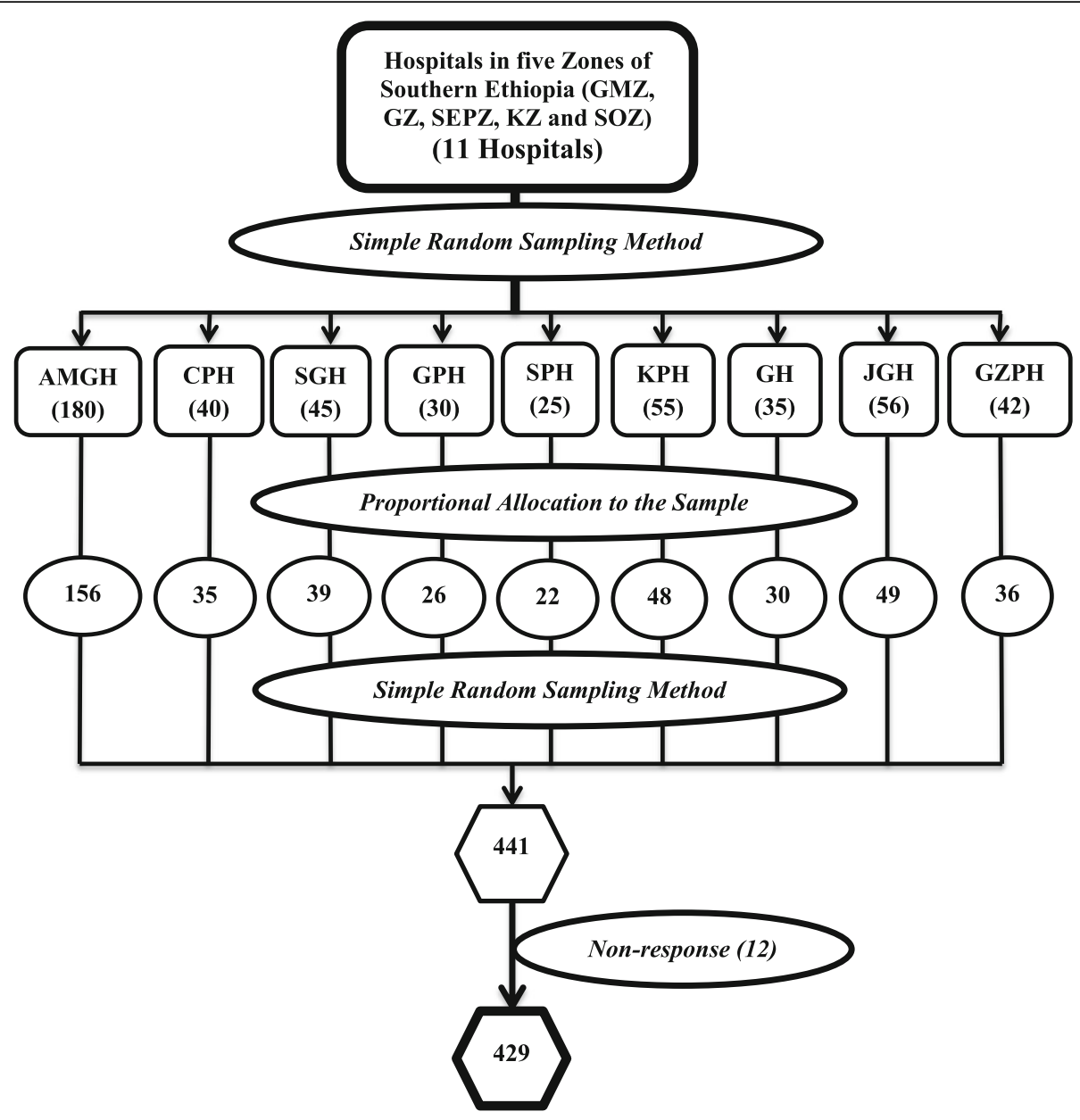

Where:- GMZ: Gamo Zone, GZ: Gofa Zone, SEPZ: Segen Areas People Zone, KZ: Konso Zone SOZ: South Omo Zone, AMGH: Arba Minch General Hospital, CPH: Chencha Primary Hospital, SGH: Sawla General Hospital, GPH: Gerese Primary Hospital, SPH: Selamber Primary Hospital, KPH: Karat Primary Hospital, GH: Gidole Primary Hospital, JGH: Jinka General Hospital and GZPH: Gazer Primary Hospital

Fig. 1 Schematic presentation of the sampling procedure for the study conducted among health professionals in public hospitals of Southern Ethiopia, 2019

Table 1 Operational definitions of variables and measurements to study conducted among health professionals in public hospitals of Southern Ethiopia, 2019

\begin{tabular}{ll}
\hline Variables & Descriptions \\
\hline Health professionals & $\begin{array}{l}\text { Health care providers (nurses, midwives, health officers, Integrated Emergency Surgery and Obstetrics (IESO) and } \\
\text { medical doctors) who were working in the delivery ward, NICU, pediatric ward and OR and have access to HBB. }\end{array}$ \\
$\begin{array}{l}\text { Knowledge of health } \\
\text { professionals on HBB }\end{array}$ & $\begin{array}{l}\text { Level of awareness of the health care providers on HBB. Knowledge of health professionals was considered } \\
\text { adequate when they answered correctly at least } 16 \text { out of } 20 \text { knowledge, assessing questions on helping babies } \\
\text { breathe (total score of } \geq 80 \% \text { ) after following algorithms for the main American Heart Association (AHA) advanced } \\
\text { cardiac life support, and inadequate when they correctly answered less than } 16 \text { out of } 20 \text { questions (total score }< \\
80 \%)[32,43] .\end{array}$ \\
Skills of health professionals & $\begin{array}{l}\text { Skills of health professionals were good for who responded correctly at least } 32 \text { of the } 40 \text { activities on skill } \\
\text { assessment tools for HBB (total score of } \geq 80 \% \text { after following algorithms for the main AHA advanced cardiac life } \\
\text { support, and poor for who correctly responded in less than } 32 \text { of the } 40 \text { activities (total score }<80 \%) ~[24,31] .\end{array}$ \\
Well-equipped facility & $\begin{array}{l}\text { Those facilities that have both essential items (mucus extractors, infant Ambu bag, face masks, towels, and } \\
\text { newborn resuscitation table) and priority items (syringes, suction apparatus, stethoscope for use with newborns } \\
\text { and source of warmth). }\end{array}$ \\
\hline
\end{tabular}




\section{Data quality control}

To maintain the validity of data to be collected randomly selected study participants were involved and standard questionnaires and checklist were used. The pre-test was conducted for inconsistencies and misunderstandings of the questions to assure reliability. Extensive training was given to data collectors and supervisors regarding the objective of the study, data collection tool, ways of data collection, checking the completeness of data collection tools and how to maintain confidentiality to maintain the quality of data to be collected. All data were checked for completeness, accuracy, clarity, and consistency by the principal investigator and by supervisors, on the day of data were collected. Data were checked for completeness before data entry into the software. Proper coding and categorization of data were maintained in the quality of the data to be analyzed.

\section{Data processing and management}

The data collectors, supervisors, and the corresponding author were in charge of verifying the fullness of the information. The data collectors filled in the date and signed each questionnaire, which was well ahead plaid, corrected and signed by the supervisors regularly. Hard copies were used to collect the data and kept in a locked cabinet by each supervisor until gathered by the principal investigator during supervision. Then, the data were coded, cleaned, edited and entered into Epi data version 3.1 to minimize logical errors and design skipping patterns, and exported to SPSS version 25 for analysis.

\section{Data analysis}

Univariate, bivariate, and multivariable analysis was done. The assumptions for binary logistic regression were checked. Hosmer-Lemeshow statistic and Omnibus tests were done for model fitness. Variables with $P<0.25$ in the bivariate analysis, variables that were significant in previous studies, and context point of view were considered to select the candidate variables for the final model. Collinearity statistics (Variance inflation factor (VIF) > 10 and tolerance $(\mathrm{T})<0.1$ were considered as suggestive of the existence of multi co-linearity. Adjusted Odds Ratio along with $95 \%$ CI was estimated to identify factors affecting health professional's skills of HBB. The $P$-value $<0.05$ was considered to declare a result as statistically significant. Then simple frequencies, summary measures, tables, and figures were used to present the information.

\section{Results}

\section{Socio-demographic characteristics of the respondents}

In this study, 429 health professionals participated, yielding a response rate of $97.3 \%$. The mean age and standard deviation of study participants were $29.7 \pm$ SD4.88 years old. More than half $(55.9 \%)$ of the study participants were females and $281(65.5 \%)$ were married. Two hundred $(46.6 \%)$ of health professionals were orthodox religion followers (Table 2).

\section{Provider related factors}

Of the total respondents, 248(57.8\%) were nurses and 213(49.7\%) were diploma holders. Two hundred fortythree $(56.6 \%)$ of health professionals were working in general hospitals. One hundred sixty-seven $(38.9 \%)$ of the health professionals had work experience of 3 to 6 years and almost half (52.0\%) had been working for 12 to 52 months in the specified wards. Out of the total study participants, 333(77.6\%) received training on $\mathrm{HBB}$ and $375(87.4 \%)$ had recent involvement in the help to baby's breath. Regarding the type of training, 135(40.6\%), $117(35.1 \%)$ and $81(24.3 \%)$ received pre, in and both pre and in-service respectively (Table 3 ).

\section{Facility related factors}

The number of deliveries occurred in facilities of Gamo, Gofa, Segen Areas People, Konso and South Omo Zone in the past 12 months was ranged from 487 to 4640 and on average 1488 deliveries. On the other hand, the number of newborn deaths occurred within $24 \mathrm{~h}$ after

Table 2 Socio-demographic characteristics of the health professionals in hospitals of Southern Ethiopia, 2019

\begin{tabular}{lll}
\hline Characteristics & Frequency & Percentage (\%) \\
\hline Age of respondent & 48 & 11.2 \\
$15-24$ & 317 & 73.9 \\
$25-34$ & 64 & 14.9 \\
$\geq 35$ & & \\
Sex & 189 & 44.1 \\
Male & 240 & 55.9 \\
Female & & \\
Marital status & 281 & 65.5 \\
Married & 17 & 4 \\
Divorced & 4 & 0.9 \\
Widowed & 127 & 29.6 \\
Single & & \\
Religion & 200 & 46.6 \\
Orthodox & 207 & 48.3 \\
Protestant & 6 & 1.4 \\
Catholic & 16 & 3.7 \\
Muslim & 68 & 59.2 \\
Salary & 254 & \\
$<3579 E T B$ & & \\
3579-5452ETB & 107 & \\
$>$ 5452ETB & & \\
\hline & &
\end{tabular}

Note: $1 E T B=0.036$ USD 
Table 3 Provider related factors of the health professionals in hospitals of Southern Ethiopia, 2019

\begin{tabular}{|c|c|c|}
\hline Variables & Frequency & Percentage (\%) \\
\hline \multicolumn{3}{|l|}{ Profession } \\
\hline Nurse & 248 & 57.8 \\
\hline Midwives & 117 & 27.3 \\
\hline Health Officers & 28 & 6.5 \\
\hline IESO & 28 & 6.5 \\
\hline Medical Doctors & 8 & 1.9 \\
\hline \multicolumn{3}{|l|}{ Qualification } \\
\hline Diploma & 213 & 49.7 \\
\hline $\mathrm{BSC}$ & 173 & 40.3 \\
\hline MSC & 35 & 8.2 \\
\hline General Practitioner & 6 & 1.4 \\
\hline Specialists $^{\oplus}$ & 2 & 0.5 \\
\hline \multicolumn{3}{|l|}{ Unit of service } \\
\hline Delivery & 146 & 34.0 \\
\hline $\mathrm{NICU}$ & 92 & 21.4 \\
\hline Pediatric Ward & 130 & 30.3 \\
\hline OR & 61 & 14.2 \\
\hline \multicolumn{3}{|c|}{ Year of experience in specified ward } \\
\hline$<12$ month & 112 & 26.1 \\
\hline $12-52$ month & 223 & 52.0 \\
\hline$>52$ month & 94 & 21.0 \\
\hline \multicolumn{3}{|c|}{ Year of experience in work } \\
\hline$<3$ year & 172 & 40.1 \\
\hline 3-6 year & 167 & 38.9 \\
\hline$>6$ year & 90 & 21.0 \\
\hline \multicolumn{3}{|l|}{ Training on $\mathrm{HBB}$} \\
\hline Yes & 333 & 77.6 \\
\hline No & 96 & 22.4 \\
\hline \multicolumn{3}{|c|}{ Recent involvement in HBB } \\
\hline Yes & 375 & 87.4 \\
\hline No & 54 & 12.6 \\
\hline
\end{tabular}

obstetrician and gynecologist, pediatrician

delivering in the past 12 months was ranging from 3 to 46 and 11 deaths on average. All the facilities were assessed for the performance of newborn resuscitation and all had performed with the minimum of one newborn's resuscitation in the past 3 months. Besides, facilities were categorized as a primary and general hospital and assessed for the availability of guidelines, essential items, priority items, and infrastructures (Table 4).

\section{Knowledge of helping babies breathe}

Regarding diagnosis of birth asphyxia, 390(90.9\%) stated depressed breathing and 356(83.0\%) indicated initial steps of newborn resuscitation were positioning the head to slightly extended neck. Four hundred fifteen (96.7\%) reported a place mask to cover chin, mouth, and nose while resuscitating with a bag and mask or tube and mask. Of the total respondents, 384(89.5\%) stated initiate breastfeeding after $30 \mathrm{~s}$ if the baby is breathing and there is no sign of respiratory difficulty. The majority (90.9\%) of the study participants stated that administer oxygen, if available and $370(86.2 \%)$ reported continue to ventilate if the newborn does not initiate breathing, or breathing is less than 30 per minute, or if there is an intercostal retraction or grunting (Table 5).

Overall, $76.2 \%$ (95\%CI: $72.2,80.3 \%$ ) of the study participants had adequate knowledge in helping babies breathe.

\section{Skills of helping babies breathe}

Of the study participants, 347(80.9\%) itemized make sure equipment is ready for use before staring resuscitating with bag and mask and 404(94.2\%) stated position to head in a slightly extended position while resuscitating by using a bag and mask. During ventilation, three hundred forty-eight (81.1\%) stated that observe chest for easy rise and fall if the newborn chest is not rising 376(87.6\%) reported that reposition mask to improve sealing. After resuscitation, if breathing is normal (no indrawing or grunting) $386(90.0 \%)$ of the study participants stated the place the baby in skin-to-skin contact with the mother and observe breathing at frequent intervals. The majority (90.0\%) stated that if newborn breathes with severe chest indrawing, ventilate with oxygen if available. Three hundred fifty-five (82.8\%) stated that disinfect mucous extractors with chemicals, and rinse all parts with clean water and allow to air dry for reusable (Table 6).

Overall, $71.1 \%$ (95\%CI: $66.8,75.4 \%)$ of the study participants had good skills on helping babies breathe.

\section{Factors associated with the skills of helping babies breathe}

Age, training on $\mathrm{HBB}$, well-equipped facility, and adequate knowledge on HBB had significantly associated with a health professional's skills of HBB after controlling for confounders in the multivariable model.

Health professionals whose ages ranged from 25 to 34 years old were 2.24 times more likely had skills on HBB as compared to the age group from 15 to 24 years old $(\mathrm{AOR}=2.24,95 \% \mathrm{CI}: 1.04,4.81)$. Those who received training on $\mathrm{HBB}$ were $69 \%$ more likely had skills on $\mathrm{HBB}(\mathrm{AOR}=2.69,95 \% \mathrm{CI}: 1.49,4.87)$. The odds of skills of $\mathrm{HBB}$ among health professionals who working in a well-equipped facility was 2.15 times $(\mathrm{AOR}=2.15$, 95\%CI: $1.09,4.25)$. Those health care providers who had adequately knowledgeable on $\mathrm{HBB}$ were 2.21 times more 
Table 4 Facility related factors for the study conducted among health professionals in public hospitals of Southern Ethiopia, 2019

\begin{tabular}{|c|c|c|c|c|}
\hline \multirow[t]{2}{*}{ Facility } & \multicolumn{2}{|c|}{ Primary Hospitals $(n=6)$} & \multicolumn{2}{|c|}{ General Hospitals $(n=3)$} \\
\hline & Frequency & $(\%)$ & Frequency & $(\%)$ \\
\hline \multicolumn{5}{|l|}{ Availability of guidelines } \\
\hline Management of newborn complications & 6 & 100 & 3 & 100 \\
\hline Postnatal care of newborns & 4 & 66.7 & 2 & 66.7 \\
\hline Immediate newborn care & 6 & 100 & 2 & 66.7 \\
\hline \multicolumn{5}{|l|}{ Availability of essential items } \\
\hline Mucus extractor & 6 & 100 & 2 & 66.7 \\
\hline Infant Ambu bag & 6 & 100 & 2 & 66.7 \\
\hline Infant face masks (sizes $0,1,2$ ) & 6 & 100 & 3 & 100 \\
\hline Towels or cloth for newborn & 6 & 100 & 2 & 66.7 \\
\hline Newborn resuscitation table & 6 & 100 & 3 & 100 \\
\hline \multicolumn{5}{|l|}{ Availability of priority items } \\
\hline Syringes ( $1 \mathrm{ml}, 2 \mathrm{ml}, 5 \mathrm{ml}, 10 \mathrm{ml}$ ) & 6 & 100 & 2 & 66.7 \\
\hline Suction apparatus & 6 & 100 & 3 & 100 \\
\hline Stethoscope for use with newborns & 4 & 66.7 & 3 & 100 \\
\hline Source of warmth & 6 & 100 & 3 & 100 \\
\hline Infrastructures $($ mean $\pm S D)$ & $50(1.17)$ & & $33.3(1.53)$ & \\
\hline
\end{tabular}

aelectricity, generator, availability of water in different parts of the facility, various kinds of telephone, radio, television, light source, ventilation, toilet, heating, fan or air conditioning, curtains for patient privacy and waiting

likely had skills on $\mathrm{HBB}(\mathrm{AOR}=2.21,95 \% \mathrm{CI}: 1.25,3.89)$ (Table 7).

\section{Discussion}

The skills of health professionals on HBB is very pivotal to save the life of many neonates during the intrapartum period and to reduce neonatal mortality at large. But, conclusive evidence was lacked to show a clear direction for intervention in many settings including our country Ethiopia. The erstwhile studies were not assessed determinates for the skills of health professionals on HBB in a comprehensive way. Therefore, this study showed the recent status of health professionals on the skills of $\mathrm{HBB}$ and factors affecting in the study setting.

In this study, $71.1 \%$ (95\%CI: $66.8,75.4 \%)$ of the study participants had good skills in HBB. Age, training on $\mathrm{HBB}$, well-equipped facility, and knowledge on HBB had significantly associated with health professionals skills on HBB.

The magnitude of good skills of health professionals on $\mathrm{HBB}$ was in line with a study done in Afghanistan (66 and 71\%) and higher than studies done in Tanzania (32.4\%), Iraq (52\%) and Ethiopia (55.8\%) [22, 24, 31, 33]. The reason for this discrepancy in the skills of HBB is because there is an advance in the health care system and refreshment pieces of training are given to health professionals by concerning bodies.

Age of the respondents ranged from 25 to 34 years old were significantly associated with the skills of HBB. But, this was incongruent with the studies done in Ethiopia $[24,32]$. This is difference may be due methodological, those health professionals whose ages were in this category were received training on $\mathrm{HBB}$, and the majority of health professionals are in this age group as from normal demography. Finding from this study showed that training on HBB, and working in well-equipped facilities were significantly associated with health professional's skills on HBB. This is in line with different studies done Kenya, Afghanistan, Rwanda, Tanzania, India, developing countries, Ethiopia, Nepal and Global Network research sites (Nagpur and Belgaum, India and Eldoret, Kenya), low income countries, resource-limited settings, Cameroon and Dominican Republic [4, 13, 17, 22-24, 26, 32, 34-40]. The reason this is due to the fact health professionals who received training as well as refreshment training has recent memory and actively performs the activities. Similarly, those providers who are working in well-equipped facilities are frequent exposure to cases and equipment used for HBB and developing their skills from time to time.

As revealed in the study knowledge of health professionals on $\mathrm{HBB}$ was significantly associated with the skills of HBB. This is consistent with studies conducted in Kenya, Cameroon, Nepal, and Ethiopia [13, 23, 26, 27]. The fact for this is knowledgeable health care professionals are more likely having the skills and perform the activities in a good manner as much as possible.

In this study profession, and years of experience working in specified wards (NICU, and maternity unit) had 
Table 5 Knowledge of helping babies breathe among health professionals in public hospitals of Southern Ethiopia, 2019

\begin{tabular}{|c|c|c|}
\hline Categories & Frequency & Percentage (\%) \\
\hline \multicolumn{3}{|l|}{ How do you diagnose birth asphyxia? } \\
\hline Depressed breathing & 390 & 90.9 \\
\hline $\mathrm{HR}<100 / \mathrm{min}$ & 354 & 82.5 \\
\hline Central cyanosis & 340 & 79.3 \\
\hline \multicolumn{3}{|l|}{ What are the initial steps of newborn resuscitation? } \\
\hline Place newborn face up & 343 & 80.0 \\
\hline Wrap or cover baby & 355 & 82.8 \\
\hline Position head so the neck is slightly extended & 356 & 83.0 \\
\hline Aspirate mouths and then nose & 342 & 79.7 \\
\hline Explain to mother what is happing & 344 & 80.2 \\
\hline \multicolumn{3}{|l|}{ What do you do when resuscitating with a bag and mask or tube and mask? } \\
\hline Place mask to cover chin, mouth, and nose & 415 & 96.7 \\
\hline Ensure seal between mask and face & 387 & 90.2 \\
\hline Ventilate 1 or 2 times and see if the chest is rising & 386 & 90.0 \\
\hline Ventilate 40 times per minute for 1 min & 359 & 83.7 \\
\hline Pause to determine whether the baby is breathing spontaneously & 356 & 83.0 \\
\hline \multicolumn{3}{|c|}{ What do you do if the baby is breathing and there is no sign of respiratory difficulty? After $30 \mathrm{~s}$} \\
\hline Keep baby warm & 383 & 89.3 \\
\hline Initiate breastfeeding & 384 & 89.5 \\
\hline Continue monitoring the baby & 379 & 88.3 \\
\hline \multicolumn{3}{|c|}{ What do you do if the baby does not begin breathing, breathing is less than 30 per minute, or if there is intercostal retraction or grunting? } \\
\hline Continue to ventilate & 370 & 86.2 \\
\hline Administer oxygen, if available & 390 & 90.9 \\
\hline Assess the need for special care & 350 & 81.6 \\
\hline Explain to mother what is happening & 350 & 81.6 \\
\hline
\end{tabular}

no significant association with the skills of $\mathrm{HBB}$. This is contradicted with the studies done in Tanzania, Kenya, and Afghanistan [21, 22, 31, 40, 41]. This is due to differences in the health care system (having the wellequipped facility, competent staff, advanced system, and quality of delivery) and methodological facets. Salary, academic qualification, year of experience in work, recent performance in work, and confidence in offering $\mathrm{HBB}$ were not significantly associated with the skills of health professionals on HBB. This is in line with the studies done in Ghana, Afghanistan, and Northwest and Northeast Ethiopia [24, 31, 42, 43]. But, incongruent with the study conducted in Eastern Ethiopia [32]. The reason for this may be methodological (having different sampling methods and procedures, study setting, and study period).

The main limitation of the study was that the study might be subjected to recall bias, the skills part was assessed by the interview that it may be their drawback. The causal association was under caution as the study design was cross-sectional. So, those limitations will be deciphered by assessing the skill part through direct observation on mannequins or if possible on real patients, and by doing follow up studies to develop cause and effect relationships.

The public health importance of this study was: The quality, and skilled care during prenatal, intrapartum, and postpartum periods are very pivotal for the health of the baby. The perinatal period is the most critical time in which most fetuses and newborns resulted in different complications. Hence, health professionals take the lion share in the health care system to avert those complications. The health professional's skill on $\mathrm{HBB}$ is very essential to saving the life of the newborns those in need. Reduction in neonatal mortality is not that much satisfactory as compared to under 5 years of child deaths. The majority of neonatal deaths are related to intrapartum complications. One of the intrapartum complications which occurred commonly is birth asphyxia that could be managed by HBB. So, identifying the frequent barriers and giving immediate interventions is very essential. 
Table 6 Skills of helping babies breathe among health professionals in public hospitals of Southern Ethiopia, 2019

\begin{tabular}{|c|c|c|}
\hline Categories & Frequency & Percentage (\%) \\
\hline \multicolumn{3}{|l|}{ Getting ready } \\
\hline Make sure equipment is ready for use & 347 & 80.9 \\
\hline Wash hands and wear gloves & 343 & 80.0 \\
\hline Quickly dry and wrap or cover the newborn & 314 & 73.2 \\
\hline Place newborn on the back on the clean, warm surface & 288 & 67.1 \\
\hline Tell women what is going to be done, listen to her, and respond to her questions and concerns & 282 & 65.7 \\
\hline Provide emotional support and reassurance & 282 & 65.7 \\
\hline \multicolumn{3}{|l|}{ Resuscitating using a bag and mask } \\
\hline Position head in the slightly extended position & 404 & 94.2 \\
\hline Suction first the mouth and then the nose & 392 & 91.4 \\
\hline Introduce catheter into mouth and suction & 372 & 86.7 \\
\hline Introduce catheter into each nostril and suction & 376 & 87.6 \\
\hline Suction well if blood or meconium is on the newborn's mouth and/or nose & 383 & 89.3 \\
\hline If the baby is still not breathing, start ventilating & 390 & 90.9 \\
\hline Recheck position of newborn's head & 373 & 86.9 \\
\hline Place the correct-sized mask on the newborn's face & 367 & 85.5 \\
\hline Form a seal between the mask and the newborn's face & 350 & 81.6 \\
\hline Squeeze bag & 338 & 78.8 \\
\hline Check the seal by ventilating and observing chest rise & 353 & 82.3 \\
\hline \multicolumn{3}{|l|}{ If the newborn's chest is rising: } \\
\hline Ventilate at 40 breaths/minute & 344 & 80.2 \\
\hline Observe chest for easy rise and fall & 348 & 81.1 \\
\hline \multicolumn{3}{|l|}{ If the newborn's chest is not rising: } \\
\hline Check the position of the head again & 367 & 85.5 \\
\hline Reposition mask to improve the seal & 376 & 87.6 \\
\hline Squeeze the bag harder; repeat suction & 361 & 84.1 \\
\hline Ventilate for $1 \mathrm{~min}$ and then assess if the newborn is breathing & 363 & 84.6 \\
\hline \multicolumn{3}{|l|}{ If breathing is normal (no indrawing or grunting): } \\
\hline Place in skin-to-skin contact with mother & 386 & 90.0 \\
\hline Observe breathing at frequent intervals & 386 & 90.0 \\
\hline Encourage the mother to begin breastfeeding & 378 & 88.1 \\
\hline \multicolumn{3}{|l|}{ If the newborn is breathing with severe indrawing: } \\
\hline Ventilate with oxygen, if available & 386 & 90.0 \\
\hline Arrange immediate transfer for special care & 354 & 82.5 \\
\hline If there is no gasping or breathing at all after 20 min of ventilation, stop ventilating & 352 & 82.1 \\
\hline \multicolumn{3}{|l|}{ Post-procedure tasks } \\
\hline Place disposable suction catheters and mucus extractors in a leak-proof container & 354 & 82.5 \\
\hline \multicolumn{3}{|l|}{ For reusable catheters and mucus extractors } \\
\hline Place in a chlorine solution for $10 \mathrm{~min}$ & 354 & 82.5 \\
\hline Wash in water and detergent & 354 & 82.5 \\
\hline Use a syringe to flush catheters/tubing & 351 & 81.8 \\
\hline Boil or disinfect in a chemical solution & 334 & 77.9 \\
\hline Take apart valve/mask and inspect for cracks/tears & 333 & 77.6 \\
\hline Wash valve/mask and check for damage & 339 & 79.0 \\
\hline Select sterilization or high-level disinfection method & 346 & 80.7 \\
\hline Wash hands and dry with a clean cloth or air entry & 350 & 81.6 \\
\hline After chemical disinfection, rinse all parts with clean water and allow to air dry & 355 & 82.8 \\
\hline
\end{tabular}


Table 7 Factors associated with the skills of helping babies breathe among health professionals in public hospitals of Southern Ethiopia, 2019

\begin{tabular}{|c|c|c|c|c|}
\hline \multirow[t]{2}{*}{ Variables } & \multicolumn{2}{|l|}{ Skills of HBB } & \multirow{2}{*}{$\begin{array}{l}\text { Crude OR } \\
95 \% \mathrm{Cl}\end{array}$} & \multirow[t]{2}{*}{ Adjusted OR } \\
\hline & Good & Poor & & \\
\hline \multicolumn{5}{|l|}{$\overline{\text { Age }}$} \\
\hline $15-24$ & $23(47.9 \%)$ & $25(52.1 \%)$ & 1 & 1 \\
\hline $25-34$ & $236(74.4 \%)$ & $81(25.6 \%)$ & $3.17(1.70,5.89)$ & $2.24(1.04,4.81)^{*}$ \\
\hline$>34$ & $46(71.9 \%)$ & $18(28.1 \%)$ & $2.78(1.27,6.09)$ & $1.92(0.68,5.39)$ \\
\hline \multicolumn{5}{|l|}{ Salary } \\
\hline$<3579$ ETB & $42(61.8 \%)$ & $26(38.2 \%)$ & 1 & 1 \\
\hline 3579-5452ETB & $174(68.5 \%)$ & $80(31.5 \%)$ & $1.35(0.77,2.35)$ & $0.90(0.43,1.89)$ \\
\hline$>5452 \mathrm{ETB}$ & $89(83.2 \%)$ & $18(16.8 \%)$ & $3.06(1.51,6.19)$ & $1.13(0.38,3.42)$ \\
\hline \multicolumn{5}{|l|}{ Profession } \\
\hline Nurse & $167(67.3 \%)$ & $81(32.7 \%)$ & $0.64(0.42,0.99)$ & $0.72(0.43,1.22)$ \\
\hline Other@ & $138(76.2 \%)$ & $43(23.8 \%)$ & 1 & 1 \\
\hline \multicolumn{5}{|l|}{ Qualification } \\
\hline Diploma & $133(62.4 \%)$ & $80(37.6 \%)$ & $0.43(0.28,0.66)$ & $0.55(0.29,1.03)$ \\
\hline Other ${ }^{\oplus}$ & $172(79.6 \%)$ & $44(20.4 \%)$ & 1 & 1 \\
\hline \multicolumn{5}{|c|}{ Year of experience in specified ward } \\
\hline$<12$ month & $60(53.6 \%)$ & $52(46.4 \%)$ & 1 & 1 \\
\hline $12-52$ month & 169 (75.8\%) & $54(24.2 \%)$ & $2.71(1.68,4.39)$ & $1.61(0.90,2.86)$ \\
\hline$>52$ month & $76(80.9 \%)$ & $18(19.1 \%)$ & $3.66(1.94,6.89)$ & $1.83(0.83,4.03)$ \\
\hline \multicolumn{5}{|c|}{ Year of experience in work } \\
\hline$<3$ year & $116(67.4 \%)$ & $56(32.6 \%)$ & 1 & 1 \\
\hline 3-6 year & 120 (71.9\%) & $47(28.1 \%)$ & $1.23(0.78,1.96)$ & $0.99(0.54,1.82)$ \\
\hline$>6$ year & $69(76.7 \%)$ & $21(23.3 \%)$ & $1.59(0.89,2.84)$ & $0.90(0.41,1.97)$ \\
\hline \multicolumn{5}{|l|}{ Training on $\mathrm{HBB}$} \\
\hline Yes & $258(77.5 \%)$ & $75(22.5 \%)$ & $3.59(2.23,5.77)$ & $2.69(1.49,4.87)^{*}$ \\
\hline No & $47(49.0 \%)$ & $49(51.0 \%)$ & 1 & 1 \\
\hline \multicolumn{5}{|c|}{ A recent performance on offering $\mathrm{HBB}$} \\
\hline Yes & $281(74.9 \%)$ & $94(25.1 \%)$ & $3.74(2.08,6.71)$ & $1.78(0.82,3.89)$ \\
\hline No & $24(44.4 \%)$ & $30(55.6 \%)$ & 1 & 1 \\
\hline \multicolumn{5}{|c|}{ Confidence in offering HBB } \\
\hline Very confident & $237(74.8 \%)$ & $80(25.2 \%)$ & $3.56(1.71,7.38)$ & $1.68(0.67,4.23)$ \\
\hline Somewhat confide. & $53(67.1 \%)$ & $26(32.9 \%$ & $2.45(1.07,5.61)$ & $1.99(0.72,5.50)$ \\
\hline Not confident & $15(45.5 \%)$ & $18(54.5 \%)$ & 1 & 1 \\
\hline \multicolumn{5}{|l|}{ Well-equipped facility } \\
\hline Yes & $277(75.3 \%)$ & $91(24.7 \%)$ & $3.59(2.06,6.26)$ & $2.15(1.09,4.25)^{*}$ \\
\hline No & 28 (45.9\%) & $33(54.1 \%)$ & 1 & 1 \\
\hline \multicolumn{5}{|l|}{ Knowledge on HBB } \\
\hline Inadequate & 47 (46.1\%) & $55(53.9 \%)$ & 1 & 1 \\
\hline Adequate & $258(78.9 \%)$ & $69(21.1 \%)$ & $4.38(2.73,7.01)$ & $2.21(1.25,3.89)^{*}$ \\
\hline
\end{tabular}

OMidwives, Health officers, IESO and medical doctors, ${ }^{\circledR} \mathrm{BSC}, \mathrm{MSc}, \mathrm{GP}$ and Specialists and *Significant at $P<0.05$

This study showed the recent status of health professionals on the skills of $\mathrm{HBB}$ and the factors affecting it. This is very important and gives direction to do an intervention on the skills of $\mathrm{HBB}$ to improve the quality of intrapartum care and to reduce neonatal mortality. 
In summary, there is a great controversy in previous studies to show the most frequent barriers that affect the skills of health care providers on HBB and rarity of information in Ethiopia that show the recent status of skills of HBB despite the improvement in neonatal health care services. Consequently, this study intended to fill those gaps. Training, wellequipped facility, and knowledge on HBB were identified as the most proximate, and modifiable factors for health professional's skills on HBB as it was supported by different previous studies. Age was also another variable identified in this study and which is not modifiable. The readers should consider the limitations of this study while interpreting the finding, and the other scholars will do more to overcome those limitations. The finding of this study gives paramount importance for health care providers to identify there major gaps and barriers to the HBB. It is also very important for policymakers and program formulators to design different strategies for saving the lives of many newborns.

\section{Conclusions}

This study indicated that health professionals' good skills in HBB were optimum. Age, training on HBB, well-equipped facility, and adequate knowledge on HBB were identified as independent factors for health professional's good skills on HBB. Interventions on strengthening strategies to address providing pre, inservice and refreshment training for health professionals on HBB and fulfill essential and priority items for health facilities that help to provide basic life support for the newborns. Different NGOs and stakeholders use this opportunity to contribute to the health care system to deliver training for health care providers and to well-equipped facilities to provide quality care. Educational institutions take the lion's share to provide quality educations for future health professionals and to well simulate and training the students before going to health care institutions. Besides, it also gives support for health care institutions by providing refreshment pieces of training, short and long term capacity building activities. The future researchers ought to do this study by supplementing the qualitative part to dig out the unreachable facts with quantitative study and it is highly recommended to use prospective study designs for a better outcome.

\section{Supplementary information}

Supplementary information accompanies this paper at https://doi.org/10. 1186/s12913-019-4772-z.

Additional file 1. Questionnaire.

Additional file $\mathbf{2}$. STROBE checklist.

\section{Abbreviations}

AHA: American Heart Association; HBB: Helping babies breathe; IESO: Integrated emergency surgery and obstetrics; NGO's: Nongovernmental organizations; NICU: Neonatal intensive care unit; OR: Operating room

\section{Acknowledgments}

The authors are glad to forward their appreciation to Arba Minch University for offering financial support to take on this research project. We would also like to extend our heartfelt gratitude to Gamo, Gofa, Segen Areas People, Konso and South Omo Zone Health Departments, CEOs and Medical directors of hospitals in each Zones for giving information and baseline data about the study area and health professionals as well as helping during data collection. Last but not least we are indebted to endeavor made by data collectors and supervisors to expedite this research process.

\section{Authors' contributions}

AM designed the study, involved in data collection, done the analysis and interpretation of the result and drafted the paper and participated in preparing all versions of the manuscript. SS, TG, ND, and $A B$ assisted in the design and the proposal development, monitored data collection, assisted during analysis and revised subsequent drafts of the paper. All authors read and approved the final manuscript.

\section{Authors' information}

Abera Mersha (BScN, MSc in Maternity and Neonatal Nursing), Department of Nursing, College of Medicine and Health Sciences, Arba Minch University, Arba Minch, Ethiopia.

Shitaye Shibiru (BSCN, MSc in Maternity and Reproductive Health Nursing), Department of Nursing, College of Medicine and Health Sciences, Arba Minch University, Arba Minch, Ethiopia.

Teklemariam Gultie (BScMw, MSc in Maternity and Reproductive Health Nursing), Department of Midwifery, College of Medicine and Health Sciences, Arba Minch University, Arba Minch, Ethiopia.

Nega Degefa (BSCN, MSc in Pediatric and Child Health Nursing), Department of Nursing, College of Medicine and Health Sciences, Arba Minch University, Arba Minch, Ethiopia.

Agegnehu Bante (BSCN, MSc in Maternity and Neonatal Nursing),

Department of Nursing, College of Medicine and Health Sciences, Arba

Minch University, Arba Minch, Ethiopia.

\section{Funding}

Arba Minch University provided funds for the data collection and stationary materials of this research work with a project grant code of Acct. No GOV1000021708902. The website of the university is www.amu.edu.et. "The funders had no role in study design, data collection, and analysis, decision to publish, or preparation of the manuscript."

\section{Availability of data and materials}

The data will not be shared to preserve participant anonymity.

\section{Ethics approval and consent to participate}

Ethical clearance was obtained from Arba Minch University, College of Medicine and Health Sciences, Institutional Research Ethics Review Board (IRB). Explanation about the purpose of the study and a letter of support was given to each Zonal health department and then to each hospital of respective Zones. For studies that were not clinical trials that involve invasive procedures, taking verbal or written consent was the standard requirement of the IRB of Arba Minch University. Hence, all the study participants were informed about the purpose of the study, their right to refuse and written and signed the voluntary consent was obtained from all study participants before recruiting the health professionals in the study. The anonymity of the participant was respected via the use of codes rather than the name of the participant and hospital name. The names of the participants were not reported on the findings of the study to ensure confidentiality.

Consent for publication

Not applicable.

Competing interests

The authors declare that they have no competing interests. 


\section{Author details}

'Department of Nursing, College of Medicine and Health Sciences, Arba Minch University, Arba Minch, Ethiopia. ${ }^{2}$ Department of Midwifery, College of Medicine and Health Sciences, Arba Minch University, Arba Minch, Ethiopia.

Received: 23 July 2019 Accepted: 22 November 2019

Published online: 09 December 2019

\section{References}

1. Dickson KE, et al. Scaling up quality care for mothers and newborns around the time of birth: an overview of methods and analyses of interventionspecific bottlenecks and solutions. BMC Pregnancy Childbirth. 2015:15.

2. Kc A, Bergström A, Chaulagain D, et al. Scaling up quality improvement intervention for perinatal care in Nepal (NePeriQIP); study protocol of a cluster-randomized trial. BMJ Glob Health. 2017;2:e000497. https://doi.org/ 10.1136/ bmjgh-2017-000497.

3. WHO. Maternal, newborn, child and adolescent health, Every Newborn Action Plan consultation 2014: World Health Organization 2014. Available at: https://www.who.int/maternal_child_adolescent/topics/newborn/enap_ consultation/en/. Accessed 24 Dec 2018.

4. Ersdal HL, Singhal N. Resuscitation in resource-limited settings. Semin Fetal Neonatal Med. 2013;18(6):373-8.

5. WHO, UNICEF. Every newborn: an action plan to end preventable newborn deaths. Geneva: World Health Organization; 2014. Available at: http://www. who.int/maternal_child_adolescent/topics/newborn/every-newborn-actionplan-draft.pdf

6. Msemo G, et al. Newborn mortality and fresh stillbirth rates in Tanzania after helping babies breathe training. Pediatrics. 2013;131(2):e353-60.

7. Samridhi G, Sarit S, Rajinder G. Assessment of newborn care corner in public health facilities of Ludhiana. India Indian Pediatrics. 2017;54.

8. Enweronu-Laryea C, et al. Basic newborn care and neonatal resuscitation: a multi-country analysis of health system bottlenecks and potential solutions. BMC Pregnancy Childbirth. 2015;15(2):S4.

9. Wyckoff MH, Aziz K, Escobedo MB, Kapadia VS, Kattwinkel J, Perlman JM, Simon WM, Weiner GM, Zaichkin JG. Part 13: neonatal resuscitation: 2015 American Heart Association guidelines update for cardiopulmonary resuscitation and emergency cardiovascular care. Circulation. 2015;132(suppl 2):S543-60,

10. Courtney Dohse. Neonatal resuscitation: definition, steps \& techniques, 2018. Available at: https://study.com/academy/lesson/neonatal-resuscitationdefinition-steps-techniques.html.

11. Pediatric Medicine/Resuscitation/Definition of Neonatal Resuscitation, 2017. Available at: https://en.wikibooks.org/wiki/Pediatric_Medicine/Resuscitation/ Definition_of_Neonatal_Resuscitation. Accessed 28 Dec 2018.

12. Erdsal $\mathrm{HL}$, et al. Early initiation of basic resuscitation interventions including face mask ventilation may reduce birth asphyxia related mortality in lowincome countries: a prospective descriptive observational study. Resuscitation. 2012;83:869-73.

13. Murila F, Obimbo MM, Musoke R. Assessment of knowledge on neonatal resuscitation amongst health care providers in Kenya. Pan Afr Med J. 2012; 11(1):78.

14. Berkelhamer SK, Kamath-Rayne BD, Niermeyer S. Neonatal resuscitation in low-resource settings. Clin Perinatol. 2016;43(3):573-91.

15. Exley Josephine $L R$, et al. Newborn resuscitation in Gombe State, Northeastern Nigeria. J Glob Health Action. 2018;8(2):020420.

16. Chu W, Hsu LL. The process of acquiring practical knowledge by emergency nursing professionals in Taiwan: a phenomenological study. J Emerg Nurs. 2011;37:126-31.

17. Subbiah N, Sarin J. Effectiveness of educational intervention on neonatal resuscitation among nursing personnel. Health Popul Perspect Issues. 2012; 35(1):36-45

18. Kerber KJ, et al. Counting every stillbirth and neonatal death to improve quality of care for every pregnant woman and her baby. BMC Pregnancy Childbirth. 2015:15.

19. Coffey P, Kak L, Narayanan I, Lockwood JB, Singhal N, Wall S, Johnson J, Schoen E: Case study: newborn resuscitation devices. Working Paper Prepared for The United Nations Commission on Life-Saving Commodities for Women and Children. 2012. http://www.everywomaneverychild.org/images/.

20. Wall SN, et al. Neonatal resuscitation in low-resource settings: What, who, and how to overcome challenges to scale up? Int I Gynaecol Obstet. 2009; 107:547-64.
21. Makene $\mathrm{CL}$, et al. Improvements in newborn care and newborn resuscitation following a quality improvement program at scale: results from a before and after study in Tanzania. BMC Pregnancy Childbirth. 2014;14(1): 381.

22. Mzurikwao CB, SKN K, Ernest Al. Factors influencing knowledge and practice on helping babies breathe among Skilled Birth Attendants in rural areas in Lake Zone in Tanzania; 2018.

23. Monebenimp F, Tenefopa M, Koh VM, Kago I. Competence of health care providers on the care of newborns at birth in a level-1 health facility in Yaoundé, Cameroon. Pan Afr Med J. 2012;11:45.

24. Gebreegziabher E, Aregawi A, Getinet H. Knowledge and skills of neonatal resuscitation of health professionals at a university teaching hospital of Northwest Ethiopia. World J Emerg Med. 2014;5(3):196-202.

25. Leader A, Cadet C, Lazala D, Roa W, Arroyo O, Jensen L. Collaborative implementation strategy for newborn resuscitation and essential care training in the Dominican Republic. Front Public Health. 2017;5:61. https:// doi.org/10.3389/fpubh.2017.00061.

26. Haile-Mariam A, Tesfaye N, Otterness C, Bailey PE. Assessing the health system's capacity to conduct newborn resuscitation in Ethiopia. Ethiop Med J. 2012;50:43-55.

27. Nelson CA, Spector JM. Newborn resuscitation capacity in Nepal. J Paediatr Child Health. 2011:47:83-6.

28. Patel J, Posencheg M, Ades A. Proficiency and retention of neonatal resuscitation skills by pediatric residents. Pediatrics. 2012;130.

29. Averting Maternal Death and Disability Program (AMDD): Needs Assessment of Emergency Obstetric and Newborn Care (EmONC): Facilitator's Guide. New York: Averting Maternal Death and Disability; 2009.

30. EPHI, FMOH, AHDD. ETHIOPIAN Emergency Obstetric and Newborn Care (EmONC) Assessment 2016, 2017. Final Report.

31. Kim YM, et al. Assessing the capacity for newborn resuscitation and factors associated with providers' knowledge and skills: a cross-sectional study in Afghanistan. BMC Pediatr. 2013;13:140.

32. Ali AS. Knowledge and practice of neonatal resuscitation and associated factors among health professionals at Jugel and Hiwot Fana specialized university hospital Harar, Eastern Ethiopia; 2015.

33. Jabir MM, Doglioni N, Fadhil T, Zanardo V, Trevisanuto D. Knowledge and practical performance gained by Iraqi residents after participation in a neonatal resuscitation program course. Acta Paediatr. 2009;98:1265-8.

34. Carlo WA, et al. Educational impact of the neonatal resuscitation program in low-risk delivery centers in a developing country. J Pediatr. 2009;154.

35. Musafili A, et al. Evaluating helping babies breathe: training for healthcare workers at hospitals in Rwanda. Acta Paediatr. 2013;102(1):e34-8.

36. Bang $A$, et al. Implementation and evaluation of the helping babies breathe curriculum in three resource-limited settings: does helping babies breathe save lives? A study protocol. BMC Pregnancy Childbirth. 2014;14(1):116.

37. Bang A, et al. Helping Babies Breathe (HBB) training: what happens to knowledge and skills over time? BMC Pregnancy Childbirth. 2016;16(1):364.

38. Kc A, et al. Evaluation of helping babies breathe quality improvement cycle (HBB-QIC) on retention of neonatal resuscitation skills six months after training in Nepal. BMC Pediatr. 2017;17(1):103.

39. Opiyo N, English M. In-service training for health professionals to improve the care of seriously ill newborns and children in low-income countries. Cochrane Database Syst Rev. 2015;5:CD007071.

40. Bhurji JK. Determination of the adherence of nurses to national neonatal resuscitation guidelines at Pumwani maternity [MMED dissertation]: University of Nairobi; 2014

41. Shikuku DN, et al. Quality of care during neonatal resuscitation in Kakamega County General Hospital, Kenya: a direct observation study. Vol. 2017; 2017. p. 12.

42. Alhassan A, et al. Knowledge and experience of neonatal resuscitation among midwives in Tamale. Vol. 2019; 2019. p. 8.

43. Biset G., Knowledge, practice and associated factors toward neonatal resuscitation among nurses and midwifes in South Wollo governmental hospitals, Northeast Ethiopia in School of Nursing and Midwives. 2018, Addis Ababa University.

\section{Publisher's Note}

Springer Nature remains neutral with regard to jurisdictional claims in published maps and institutional affiliations. 